\title{
N2-3 Stage Nasopharyngeal Carcinoma Patients With Cervical Node Necrosis And Extracapsular Spread Benefit From Capecitabine Maintenance Chemotherapy
}

Ting Liu ( 201070176@163.com)

Liuzhou people's Hospital https://orcid.org/0000-0003-4446-0407

Siwen Liu

liuzhou people's hospital

Yuting Gu

Liuzhou people's Hospital

Xuehua Wen

liuzhou people's hospital

Research

Keywords: nasopharyngeal carcinoma, intensity-modulated radiotherapy, chemotherapy, capecitabine, prognosis

Posted Date: July 27th, 2020

DOI: https://doi.org/10.21203/rs.3.rs-44304/v1

License: (c) (i) This work is licensed under a Creative Commons Attribution 4.0 International License.

Read Full License 


\section{Abstract}

Purpose: The purpose of this study was to analyze the efficacy and toxicities of concurrent chemoradiotherapy plus capecitabine maintenance chemotherapy for N2-3 stage nasopharyngeal carcinoma (NPC) with cervical node necrosis (CNN) and extracapsular spread(ECS).

Patients and methods: This study included 283 N2-3 stage NPC patients with CNN and extracapsular spread who were treated with concurrent chemoradiotherapy. The prescribed doses delivered to the planning target volumes (PTVs), PTVnx, PTVnd, PTV1, and PTV2, were 68-72, 66-70, 60-64, and 52-56 Gy, respectively, in 30-32 fractions. All patients received induction chemotherapy followed by concurrent chemoradiotherapy with cisplatin alone $\left(80-100 \mathrm{mg} / \mathrm{m}^{2}\right.$, day 1 , every 21 days is a cycle).Two weeks after completion of concurrent chemoradiotherapy, oral capecitabine (twice daily after meals for 14 consecutive days every 3 weeks) was administered to maintenance chemotherapy patients. Maintenance chemotherapy was administered for 6 months.

Results: The 5-year overall survival (OS), local relapse-free survival (LRFS), and distant metastasis-free survival (DMFS) rates were $84.8 \%, 95.4 \%$, and $86.6 \%$, respectively, for all patients. The 5 -year OS rates for patients who accepted capecitabine maintenance chemotherapy (group 1) and patients without maintenance chemotherapy (group 2) were $93.8 \%$ and $82.1 \%(P=0.021)$, respectively. The 5-year LRFS rates for groups 1 and 2 were $96.9 \%$ and $95.0 \%(P=0.467)$, respectively. The 5-year DMFS rates for groups 1 and 2 were $93.8 \%$ and $84.4 \%(P=0.044)$, respectively. Hand-Foot syndrome, myelotoxic effects, fatigue, and gastrointestinal symptoms were the common but not severe (grades 1-2) toxicities of the capecitabine maintenance chemotherapy.

Conclusion: Capecitabine maintenance chemotherapy improves the outcome and reduces the occurrence of distant failure in N2-3 stage NPC patients with CNN and extracapsular spread. The side effects of treatment were generally tolerated. More clinical trials are required to evaluate the efficacy and toxicity of it.

\section{Introduction}

More than 120,000 cases of nasopharyngeal carcinoma (NPC) were newly diagnosed in 2018 , with > 70,000 resulting in death. NPC is a unique carcinoma with racial and geographic variation; southwest China has the highest incidence worldwide. ${ }^{1}$ Patients with NPC are asymptomatic for a long time, and up to $85 \%$ have lymph node metastases at the time of diagnosis. ${ }^{2-3}$ Cervical node necrosis (CNN) and extracapsular spread (ECS), the incidence of which is $22.5-42.1 \%$ in patients with NPC, have previously been reported as a strong, independent negative prognostic factor for overall survival (OS), disease-free

survival (DFS), and distant metastasis-free survival (DMFS). ${ }^{4-5}$ Given the poor prognosis, it is important to find effective strategies to improve the survival outcomes of this specific sub-group of NPC patients. 
NPC is a radiosensitive malignancy. Radiotherapy forms the basis of all treatment stages, and distant metastases are the main reason for treatment failure. ${ }^{6-7}$ Many scholars are of the opinion that chemotherapy intensity should be increased to improve patients' outcomes. ${ }^{8-9}$ Nevertheless, the role of maintenance chemotherapy is controversial and increases the complication rate.

In recent years, oral 5-FU has been shown to yield very satisfactory results in multiple solid tumors. ${ }^{10-11}$ Stockler ${ }^{12}$ and Simkens ${ }^{13}$ concluded that oral capecitabine is safe and effective, and maintenance treatments with capecitabine are particularly suitable for the overall management of advanced breast and metastatic colorectal cancers. Capecitabine is an oral anticancer fluorouracil derivative that can be converted to 5-FU in vivo. The metronomic use of capecitabine may also be a good choice for NPC, considering its low toxicity and high efficacy.

The aim of the current study was to determine whether N2-3 stage NPC with a CNN and ECS treatment is optimized by concurrent chemoradiotherapy plus capecitabine maintenance chemotherapy.

\section{Material And Methods}

\section{Patients}

Between 1 January 2009 and 30 December 2015, a total of 283 patients with NPC (N2-3) and CNN and ECS were enrolled in the present study.

\section{Pretreatment evaluation}

The essential pretreatment assessments included complete medical histories, physical examinations, nasopharyngeal fiberoptic endoscopies, magnetic resonance imaging (MRI) scans of the head and neck, chest radiographies or CTs, abdominal region ultrasonographies or CTs, bone emission CT scans, hematological and biochemical profiles, and dental evaluations. All of the patients were required to provide written informed consents.

\section{Intensity-modulated radiation therapy}

Computed tomography contrast-enhanced scanning was applied from the skull cap to $2 \mathrm{~cm}$ below the clavicle, with a layer thickness of $3 \mathrm{~mm}$. Under the guidance of Report 50 and Report 62 of the International Commission on Radiation Units and Measurements, the gross tumor volume (GTV)nx and GTVnd included the entire macroscopic tumor defined after a correlative analysis by the CT and MRI scans. For the clinical target, the high-risk clinical target volume (CTV1) was defined as the nasopharynx GTV plus a 5-10 mm margin (2-3 mm posteriorly if adjacent to the brainstem or spinal cord) that can encompass the high-risk sites of microscopic extension and the whole nasopharynx. The low-risk CTV2 was defined as the nasopharynx GTV plus a 5-10 mm margin (2-3 mm posteriorly if adjacent to the brainstem or spinal cord) that can encompass the low-risk sites of microscopic extension, including the skull base, clivus, sphenoid sinus, parapharyngeal space, pterygoid fossae, posterior parts of the nasal 
cavity, pterygopalatine fossae, retropharyngeal nodal regions, and cervical node levels II, III, IV, and V. The total doses were prescribed to the median of the target volume, and the $95 \%$ isodose was approximately similar to the planning target volume (PTV). PTVnx, PTVnd, PTV1, and PTV2 were generated by adding 5mm margins to GTVnx, GTVnd, CTV1, and CTV2, respectively. The prescribed doses delivered to PTVnx, PTVnd, PTV1, and PTV2 were 68-72,66-70, 60-64, and 52-56 Gy, respectively, in 30-32 fractions.

\section{Magnetic resonance imaging diagnostic criteria}

On a MRI, the CNN is seen as a focal area of high signal intensity on T2-weighted images and as an area of low signal intensity on contrast material-enhanced T1-weighted images-with or without a surrounding rim of enhancement ${ }^{14-16}$ (Fig. 1).

ECS is defined as the presence of indistinct nodal margins, irregular nodal capsular enhancements, or the infiltration of surrounding fats or muscle planes or fusions with adjacent LNs ${ }^{17}$ (Fig.1).

\section{Chemotherapy}

All patients underwent induction chemotherapy followed by concurrent chemoradiotherapy with cisplatin alone (80-100 mg/m2, day 1 , every 21 days is a cycle).

Two weeks after the completion of concurrent chemoradiotherapy, oral capecitabine (twice daily after meals for 14 consecutive days every 3 weeks) was administered to maintenance chemotherapy patients. The dose of capecitabine was determined on the basis of the body surface area $\left[1250 \mathrm{mg} / \mathrm{m}^{2} \mathrm{twice}\right.$ per day]). Maintenance chemotherapy was administered for 6 months or terminated if there was disease progression or intolerable toxicity. The dose of capecitabine was modified according to the toxicity profile. The chemotherapy was not delayed or paused until the nadir values were $\geq 1,500$ cells/ $\mu \mathrm{L}$ for neutrophils and $\geq 100,000$ cells $/ \mu \mathrm{L}$ for platelets.

\section{Follow-up}

During the treatment, all patients underwent weekly examinations. Then, the patients were evaluated for tumor responses through nasopharyngeal fiberoptic endoscopy and MRI. Toxicities were observed and noted according to the Toxicity Criteria of the Radiation Therapy Oncology Group. Efficacy was determined using the RECIST solid tumor efficacy evaluation criteria.

Subsequently, the patients were followed up every 3 months during the first 2 years, every 6 months for the third year, and every 1 year thereafter. Each follow-up included a physical examination, nasopharyngeal fiberoptic endoscopy, chest radiography or CT, abdominal region ultrasonography or CT, and a hematological and biochemical profile. The MRI scan was performed every 6 months.

Additional CT and bone emission CT scans were performed to confirm any suspicious lesions for distant metastasis. 


\section{Statistical analysis}

The data was processed using SPSS 23.0 software. The OS was calculated from the date of diagnosis until death or the last follow-up using the Kaplan-Meier method, and the difference was analyzed using a two-sided log-rank test. The local relapse-free survival (LRFS) and DMFS were defined as the time from the day of diagnosis to the locoregional progression and distant metastasis, respectively. The LRFS and DMFS were also calculated and constructed using the Kaplan-Meier method. A comparison between the groups for the frequency of data was carried out using the $\chi 2$-test. P-values $<0.05$ were considered statistically significant.

\section{Results}

\section{Patient demographics}

The male-to-female ratio was 209:74. All patients were WHO II with respect to histologic classification. Sixty-five patients (23.0\%) accepted the capecitabine maintenance chemotherapy. The clinical characteristics of the 283 NPC patients are listed in Table 1.

\section{OS}

The 5-year OS was $84.8 \%$ for all patients. Forty-three patients died (distant metastasis, 31; locoregional recurrence, 11; and unknown cause, 1 ).

The 5-year OS rates for the patients who accepted capecitabine maintenance chemotherapy (group 1) and patients who did not accept maintenance chemotherapy (group 2) were $93.8 \%$ and $82.1 \%(P=0.021$, Figure 2), respectively.

\section{Local relapse-free survival (LRFS)}

The 5-year LRFS was $95.4 \%$. Thirteen patients exhibited locoregional recurrences. The 5-year LRFS rates for patients who accepted the capecitabine maintenance chemotherapy (group 1) and patients who did not accept the maintenance chemotherapy (group 2) were $96.9 \%$ and $95.0 \%$, respectively $(P=0.467$, Figure $3)$.

\section{DMFS}

The 5-year DMFS rate was $86.6 \%$. Thirty-eight patients presented distant metastases (lung, 8; bone, 6; liver, 9; brain, 1; soft tissue, 1; and multiple site metastases, 12).

The 5-year DMFS rates for patients who accepted the capecitabine maintenance chemotherapy (group 1) and patients who did not accept the maintenance chemotherapy (group 2) were $93.8 \%$ and $84.4 \%$, respectively $(P=0.044$, Figure 4$)$. 
Hand-Foot syndrome, myelotoxic effects, fatigue, and gastrointestinal symptoms were the common but not severe (grades 1-2) toxicities of the capecitabine maintenance chemotherapy. No grade 4 toxicity or treatment-related deaths occurred during the capecitabine maintenance chemotherapy; detailed information is covered in Table 2.

\section{Discussion}

NPC is a unique carcinoma with racial and geographical variations; southwest China has the highest incidence of NPC worldwide. ${ }^{1}$ NPC is a radiosensitive malignancy. Radiotherapy forms the basis for all treatments in patients with NPC. Intensity-modulated radiation therapy (IMRT) has replaced conventional two-dimensional radiotherapy as the pivotal radiotherapy technique, and it has improved the local control rate to $>90 \%$. Distant metastases are the main treatment failure pattern in patients with NPC. ${ }^{18-19}$ Therefore, it is crucial to choose a proper therapeutic modality to maximally reduce distant metastases.

It is unknown if increasing the intensity of chemotherapy will improve the outcome of patients with NPC Multiple studies have failed to demonstrate a significant advantage in the entire population without risk stratification. Several randomized studies have attempted to elucidate this matter, but they failed to get the expected result. ${ }^{20-21}$ In our opinion, the major goal of maintenance chemotherapy in patients with NPC is to reduce the occurrence of distant failure; however, not all NPC patients require maintenance chemotherapy. Of note, none of the studies focused on the ECS and CNN sub-groups. Due to a lack of stratification, we were intent on identifying the specific sub-group that would benefit from maintenance chemotherapy.

CNN and ECS is a late biological event in the evolution of tumor metastases in lymph nodes, and it often occurs at the interface between necrotic areas and well-aerated cells. An increase in the radioresistance of hypoxic tumor cells has been well-documented in previous studies, ${ }^{22-23}$ therefore, CNN may serve as a marker for hypoxia. ECS may serve as a marker for an increase in the number of metastases. The presence of CNN and ECS in tumors induces relatively poor sensitivity to radiotherapy or chemotherapy. Moreover, it can lead to gene amplification with a resistance that can increase tumor aggressiveness and accelerate progression. ${ }^{24-27}$

CNN and ECS proved to be an independent negative prognostic factor for OS, DFS, and DMFS. ${ }^{28-30}$ It has been reported that an increase in chemotherapy intensity provides survival benefits for high-risk patients. ${ }^{31-32}$ Therefore, identifying patients as being high risk is significant for selecting an individualized therapy strategy. Assuming that the last fraction of IMRT is completed, the tumor does not often completely regress, especially in patients with CNN and ECS. ${ }^{33}$ Thus, maintenance chemotherapy is an effective method to improve treatment outcomes.

Capecitabine is an oral pro-drug of 5-fluorouracil (5-FU), and it is frequently used for treating cancer. Capecitabine proved to have very satisfactory results in multiple solid tumors. ${ }^{11-13}$ It is completely and rapidly absorbed after oral administration. ${ }^{34-35}$ It has been concluded that oral capecitabine is safe and 
effective, and maintenance treatment with capecitabine is particularly suitable for the overall management of advanced breast cancer. ${ }^{36-37}$

In our study, the 5-year OS, LRFS, and DMFS rates were $84.8 \%, 95.4 \%$, and $86.6 \%$, respectively. The data is similar to other studies. ${ }^{28-29}$ Our data reveals the pattern of treatment failure is distant metastasis. The most common site of metastasis includes the lungs, brain, liver and bones. The 5-year DMFS rates for groups 1 and 2 were $93.8 \%$ and $84.4 \%(P=0.044)$, respectively. Thus, capecitabine maintenance chemotherapy may improve DMFS rates in N2-3 NPC patients with CNN and ECS. The role of adjuvant chemotherapy is controversial. Our study demonstrates a different conclusion compared to the previous studies. Because the shortcoming was a lack of stratification in the previous studies, ${ }^{20-21}$ we were intent on identifying the specific sub-group that would benefit from maintenance chemotherapy.

Based on our sub-group analysis, 166 patients had MRI scans of the head and neck immediately when concurrent chemoradiotherapy was completed. There were only 38 patients who had complete responses; the remaining patients had partial responses. We will choose to observe and evaluate these 3 months later. With time, the residual tumors became disintegrated and fully disappeared by 3 months' time. Our studies failed to demonstrate a significant relationship between the patients with PR and CR. The number of cases was too small to analyze statistically, a larger sample size is needed to verify this.

Two of the major constraints for maintenance chemotherapy are toxicity and tolerability. The major toxicities encountered included manageable myelosuppression, oral mucositis, and radiation dermatitis after CCRT. Patients can tolerate an oral chemotherapy within two weeks after CCRT. Hand-Foot syndrome $(n=52,80 \%)$, leucopenia $(n=45,69.2 \%)$, anemia $(n=41,63.1 \%)$, fatigue $(n=49,75.4 \%)$, and nausea $(n=39,60.0 \%)$ were the common but not severe symptoms (grades $1-2)$ during maintenance chemotherapy.

We proposed a new idea for selecting a better individualized therapy to maximally reduce distant metastases. Nevertheless, there were some limitations in this study. Specifically, the study had a retrospective design and some existent biases. Additional studies with a larger sample size and unit participation are imperative.

\section{Conclusion}

In this study we showed that capecitabine maintenance chemotherapy can improve the outcomes of and reduce the occurrence of distant failures in N2-3 stage NPC patients with CNN and ECS.

\section{List Of Abbreviations}

NPC:nasopharyngeal carcinoma; CNN:cervical node necrosis; ECS:extracapsular spread; PTVs:planning target volumes; OS:overall survival; LRFS:Iocal relapse-free survival; DMFS: distant metastasis-free survival; MRI:magnetic resonance imaging; GTV:the gross tumor volume; CTV: clinical target volume; 


\section{Declarations}

\section{Ethics approval and consent to participate}

This study was reviewed and approved by the Ethics Committee of LiuZhou People's Hospital, GuangXi, China.() Written informed consent was obtained from each patient. this study was conducted in accordance with the Declaration of Helsinki.

\section{Consent for publication}

Not applicable.

\section{Availability of data and materials}

All data generated or analyzed during this study are included in this published article.

\section{Competing interests}

The authors declare that they have no competing interests.

\section{Funding}

None.

\section{Authors' contributions}

Tingliu and Siwen liu conceived and designed the study. Yuting Gu and Xuehua Wen collected, analyzed, and interpreted the data, prepared the draft and gave final approval of the version to be submitted. Tingliu and Siwen liu undertook data analysis and interpretation, and performed the statistical analysis. All authors approved the final manuscript.

\section{Acknowledgements}

\section{References}

1. Freddie Bray, BSc, MSc, PhD; Jacques Ferlay, ME, et al. Global Cancer Statistics 2018: GLOBOCAN Estimates of Incidence and Mortality Worldwide for 36 Cancers in 185 Countries.CA CANCER J CLIN 201 8;68:394-424.

2. Xing-Li Yang, Yan Wang, et al.Additional Cervical Lymph Node Biopsy is Not a Significant Prognostic Factor for Nasopharyngeal Carcinoma in the Intensity-Modulated Radiation Therapy Era: A Propensity Score-matched Analysis from an Epidemic Area.Journal of Cancer 2018, Vol. 9

3. Ho Francis $\mathrm{C} \mathrm{H}$,Tham Ivan $\mathrm{W} \mathrm{K}_{\text {, }}$ et al.Patterns of regional lymph node metastasis of nasopharyngeal carcinoma: a meta-analysis of clinical evidence.[J]. BMC cancer,2012,12:98. 
4. Wang XS, Hu CS, Ying HM, et al. Patterns of retropharyngeal node metastasis in nasopharyngeal carcinoma. Int J Radiat Oncol Biol Phys 2009; 73(1): 194-201.

5. Lu-Lu Zhang, Jia-Xiang Li,et al.Influence of Cervical Node Necrosis of Different Grades on the Prognosis of Nasopharyngeal Carcinoma Patients Treated with Intensity-Modulated Radiotherapy.Journal of Cancer 2017, Vol. 8

6. Pfister DG, Spencer S, et al. Head and neck cancers, version1.2015. J Natl Compr Canc Netw 2015;13:847-855; quiz 856.

7. Gooi Zhen,Richmon Jeremy,et al. AHNS Series - Do you know your guidelines? Principles of treatment for nasopharyngeal cancer: A review of the National Comprehensive Cancer Network guidelines.[J]. Head \& neck,2017,392:.

8. Liu Yi-Chun,Wang Wen-Yi,et al. Prognostic impact of adjuvant chemotherapy in high-risk nasopharyngeal carcinoma patients.[J]. Oral oncology,2017,64:.

9. Jingfeng Zong, Hanchuan Xu,et al. Maintenance chemotherapy using S-1 following definitive chemoradiotherapy in patients with N3 nasopharyngeal carcinoma.Radiation Oncology (2019) 14:182.

10. Wen Linchun,You Chuanwen,et al. Phase II trial of concurrent chemoradiotherapy with S-1 versus weekly cisplatin for locoregionally advanced nasopharyngeal carcinoma.[J]. Molecular and clinical oncology,2015,33:687-691.

11. Varshavsky-Yanovsky Asya N,Goldstein Lori J,et al. Role of Capecitabine in Early Breast Cancer.[J]. Journal of clinical oncology ,2020,383:.

12. Stockler Martin R,Harvey Vernon J,et al.Capecitabine versus classical cyclophosphamide, methotrexate, and fluorouracil as first-line chemotherapy for advanced breast cancer.[J]. Journal of clinical oncology $, 2011,2934$ :.

13. Simkens LHJ, Tinteren HV, May A, et al. Maintenance treatment with capecitabine and bevacizumab in metastatic colorectal cancer(CAIRO3): a phase 3 randomised controlled trial of the Dutch Colorectal Cancer Group. Lancet 2015;385(9980):1843-1852.

14. Lan Mei,Huang Ying,et al. Prognostic Value of Cervical Nodal Necrosis in Nasopharyngeal Carcinoma: Analysis of 1800 Patients with Positive Cervical Nodal Metastasis at MR Imaging.[J]. Radiology,2015,2762:.

15. Iv jun,et al.magnetic resonance imaging analysis of regional lymph node metastasis in 1298 cases of nasopharyngeal carcinoma.J Clin otorhinolaryngol head neck surg(china).2012.vol18.

16. Guo Qiaojuan,Pan Jianji,et al.Suggestions for lymph node classification of UICC/AJCC staging system: a retrospective study based on 1197 nasopharyngeal carcinoma patients treated with intensity-modulated radiation therapy.[J]. Medicine,2015,9420:.

17. Zhou Xin,Ou Xiaomin,et al. Quantitative Metastatic Lymph Node Regions on Magnetic Resonance Imaging Are Superior to AJCC N Classification for the Prognosis of Nasopharyngeal Carcinoma.[J]. Journal of oncology,2018,2018:. 
18. Kang Min,Zhou Pingting,et al. A novel N staging system for NPC based on IMRT and RTOG guidelines for lymph node levels: Results of a prospective multicentric clinical study.[J]. Oncology letters,2018,161:.

19. Liu Ting,Lv Jun,Qin Yutao. Standardized tumor volume: an independent prognostic factor in advanced nasopharyngeal carcinoma.[J]. Oncotarget,2017,841:.

20. Chi KH, Chang YC, Guo WY, et al. A phase III study of adjuvant chemotherapy in advanced nasopharyngeal carcinoma patients. Int J Radiat Oncol Biol Phys. 2002; 52:1238-1244.

21. Chen L, Hu CS, Chen XZ, et al. Concurrent chemoradiotherapy plus adjuvantchemotherapy versus concurrent chemoradiotherapy alonein patients with locoregionally advanced nasopharyngeal carcinoma: a phase 3 multicentre randomised controlled trial. Lancet Oncol. 2012; 13:163-171.

22. Don DM, Anzai Y, Lufkin RB, et al. Evaluation of cervical lymph node metastases in squamous cell carcinoma of the head and neck.Laryngoscope 1995; 105(7 Pt 1):669-74.

23. Som P. Lymph nodes of the neck. Radiology. 1987; 165(3):593-600.

24. Toustrup K, Sorensen BS, Lassen P, et al. Gene expression classifier predicts for hypoxic modification of radiotherapy with nimorazole in squamous cell carcinomas of the head and neck. Radiother Oncol.2012;102:122-129.

25. Toustrup K, Sorensen BS, Nordsmark M, et al. Development of a hypoxia gene expression classifier with predictive impact for hypoxic modification of radiotherapy in head and neck cancer.Cancer Res. 2011;71:5923-5931.

26. Tao Ya,Wang Kun,Chen Zhen,et al.Correlation of five secretory proteins with the nasopharyngeal carcinoma metastasis and the clinical applications.[J]. Oncotarget,2017,817:.

27. Yang Gui,Deng Qiaoling,et al.Cyclooxygenase-2 expression is positively associated with lymph node metastasis in nasopharyngeal carcinoma.[J]. PloS one,2017,123:.

28. Zhang Lu-Lu,Zhou Guan-Qun,et al.Combined prognostic value of pretreatment anemia and cervical node necrosis in patients with nasopharyngeal carcinoma receiving intensity-modulated radiotherapy: A large-scale retrospective study.[J]. Cancer medicine,2017,612:.

29. Lan Mei,Chen Chunyan,et al.Neoadjuvant chemotherapy followed by concurrent chemoradiotherapy versus concurrent chemoradiotherapy alone in nasopharyngeal carcinoma patients with cervical nodal necrosis.[J]. Scientific reports,2017,7:.

30. Feng Yanru,Cao Caineng,et al.Prognostic Value and Staging Classification of Lymph Nodal Necrosis in Nasopharyngeal Carcinoma after Intensity-Modulated Radiotherapy.[J]. Cancer research and treatment ,2019,513:

31. Yao JiJin,Jin YaNan,et al.Do all patients with advanced N-stage nasopharyngeal carcinoma benefit from the addition of induction chemotherapy to concurrent chemoradiotherapy?[J]. Therapeutic advances in medical oncology,2019,11:

32. Sun Y, Li WF, Chen NY, et al. Induction chemotherapy plus concurrent chemoradiotherapy versus concurrent chemoradiotherapy alone in locoregionally advanced nasopharyngeal carcinoma: a phase 3, multicentre, randomised controlled trial. Lancet Oncol.2016,1711:. 
33. Sai Lan Liu, Lin Quan Tang,et al.The prognosis of neck residue nasopharyngeal carcinoma (NPC) patients: results from a case-cohort study.[J]. Journal of Cancer,2018,910:

34. Judson, I. R. et al. A human capecitabine excretion balance and pharmacokinetic study after administration of a single oral dose of 14C-labelled drug[J]. Investigational new drugs, 1999,171:

35. Reigner B,Blesch K,et al.Clinical pharmacokinetics of capecitabine.[J]. Clinical pharmacokinetics,2001,402:.

36. .Martin R.Stockler,Vernon J.Harvey,et al.Capecitabine versus classical cyclophosphamide, methotrexate, and fluorouracil as first-line chemotherapy for advanced breast cancer.[J]. Journal of clinical oncology $, 2011,2934$ :

37. N. Masuda, S.-J. Lee, S. Ohtani,et al. Adjuvant Capecitabine for Breast Cancer after Preoperative Chemotherapy.The New England Journal of Medicine.June 1, 2017.

\section{Tables}


Table 1

Clinical characteristics of patients

\begin{tabular}{|c|c|c|}
\hline Characteristic & CCRT-MC Patients $(n=65)$ & CCRT-non-MC Patients $(n=218)$ \\
\hline \multicolumn{3}{|l|}{ Age (years) } \\
\hline Median & 45 & 47 \\
\hline Range & $30-69$ & $20-72$ \\
\hline \multicolumn{3}{|l|}{ Gender, n (\%) } \\
\hline Male & $51(78.4)$ & $158(72.4)$ \\
\hline Female & $14(21.5)$ & $60(27.5)$ \\
\hline \multicolumn{3}{|l|}{ Histology } \\
\hline WHO II & 65 & 218 \\
\hline \multicolumn{3}{|l|}{ T stage, n (\%) } \\
\hline T1 & $1(1.5)$ & $5(2.2)$ \\
\hline T2 & 12(18.4) & 41(18.8) \\
\hline T3 & $24(36.9)$ & $96(44.0)$ \\
\hline T4 & $28(43.0)$ & $76(34.8)$ \\
\hline \multicolumn{3}{|l|}{ N stage, n (\%) } \\
\hline N2 & $48(73.8)$ & $147(67.4)$ \\
\hline N3 & $17(26.1)$ & $71(32.5)$ \\
\hline \multicolumn{3}{|l|}{ UICC stage } \\
\hline III & $28(43.0)$ & $103(47.2)$ \\
\hline IV & $37(56.9)$ & $115(52.7)$ \\
\hline \multicolumn{3}{|c|}{ NeoCT (cycles), n (\%) } \\
\hline 1 & 0 & $1(0.4)$ \\
\hline 2 & $53(82.8)$ & 187(85.3) \\
\hline 3 & 11(17.1) & $31(14.3)$ \\
\hline \multicolumn{3}{|c|}{ post-CCRT MRI response, $\mathrm{n}$} \\
\hline $\mathrm{CR}$ & 9 & 29 \\
\hline PR & 36 & 92 \\
\hline
\end{tabular}


Table 2

Adverse events during maintenance chemotherapy $(n=65)$.

\begin{tabular}{|lcccccc|}
\hline Toxicity & \multicolumn{2}{c}{ Grade 1 } & \multicolumn{2}{c|}{ Grade 2 } & \multicolumn{2}{c|}{ Grade 3 } \\
\cline { 2 - 7 } & $\mathbf{n}$ & $\%$ & $\mathbf{n}$ & $\%$ & $\mathbf{n}$ & $\%$ \\
\hline Hand-Foot syndrome & 36 & 55.4 & 16 & 24.6 & 0 & 0 \\
myelotoxic effects & & & & & & \\
\hline Leucopenia & 38 & 58.4 & 7 & 10.8 & 0 & 0 \\
\hline Anemia & 34 & 52.3 & 7 & 10.8 & 1 & 1.5 \\
\hline Thrombocytopenia & 11 & 16.9 & 0 & 0 & 0 & 0 \\
\hline fatigue & 47 & 72.3 & 2 & 3.1 & 0 & 0 \\
\hline gastrointestinal symptoms & & & & & \\
\hline Nausea & 35 & 53.8 & 4 & 6.2 & 0 & 0 \\
\hline Vomiting & 7 & 10.8 & 0 & 0 & 0 & 0 \\
\hline Diarrhea & 4 & 6.2 & 0 & 0 & 0 & 0 \\
\hline Hepatoxicity & 2 & 3.1 & 0 & 0 & 0 & 0 \\
\hline
\end{tabular}

Figures 


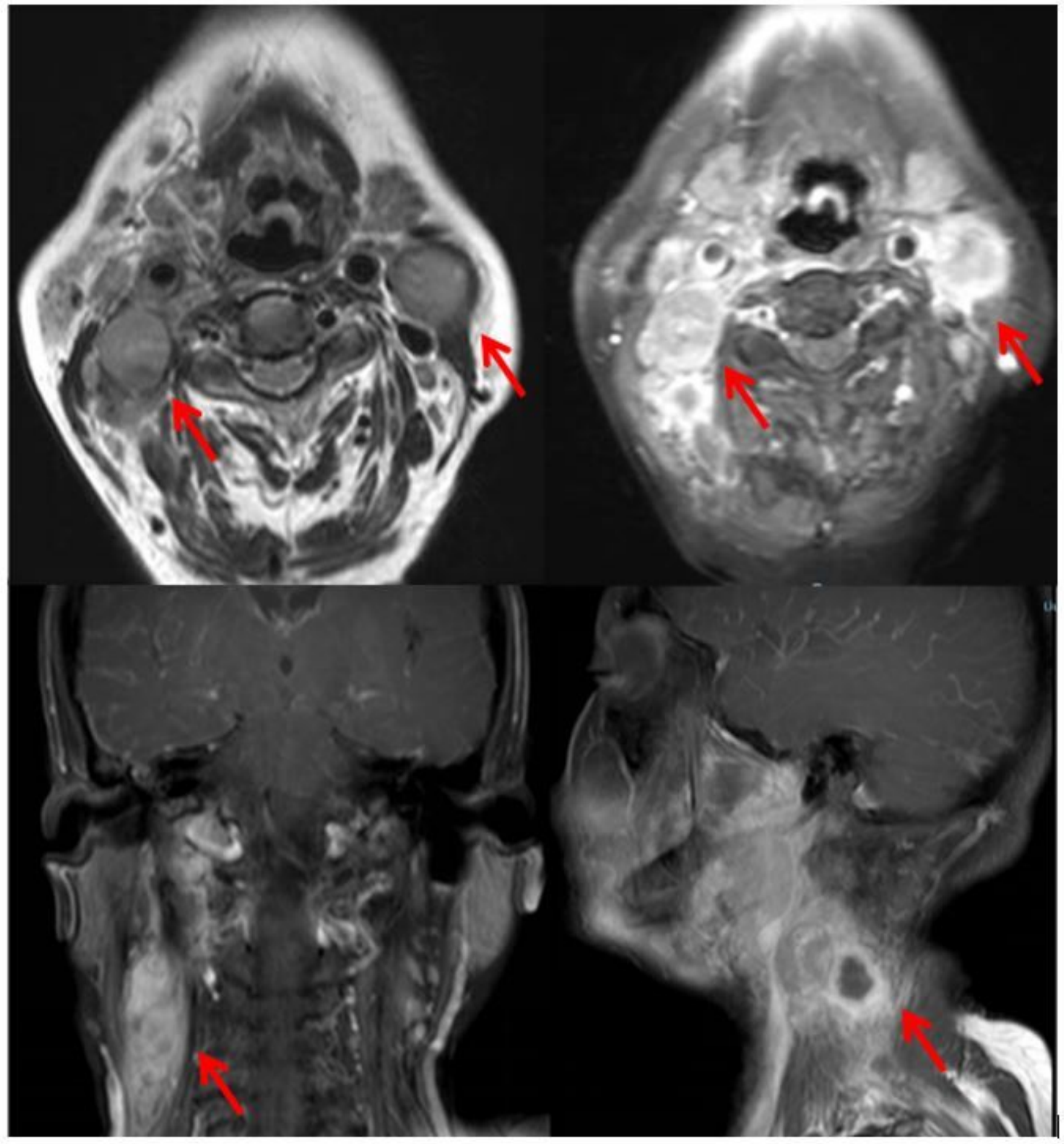

Figure 1

Representative MRI photographs of CNN and ECS 


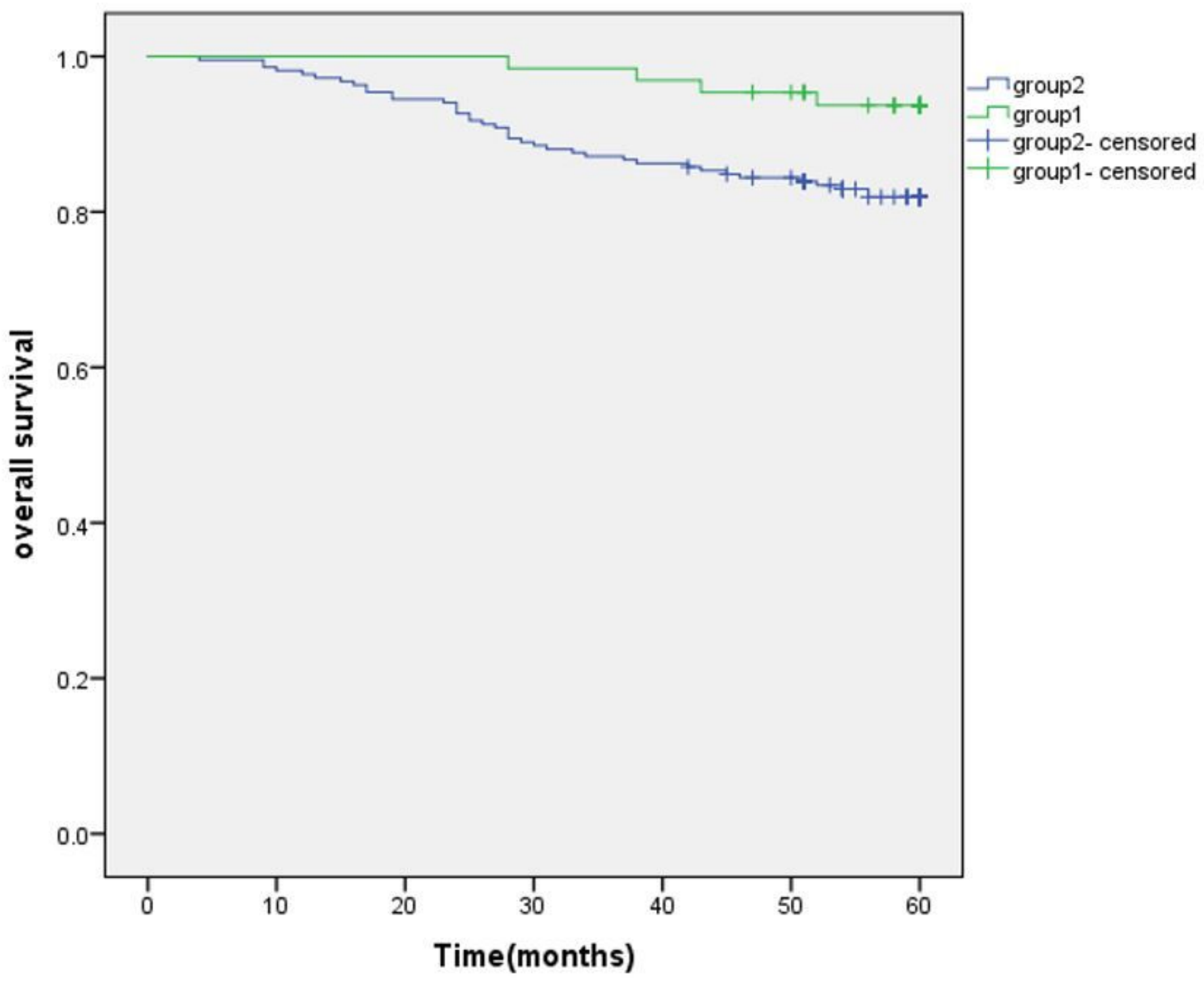

Figure 2

Kaplan-Meier estimate of overall survival for patients who accepted capecitabine maintenance

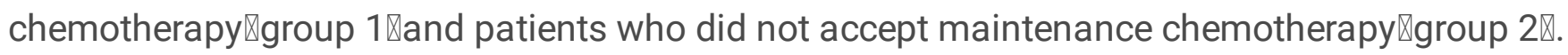




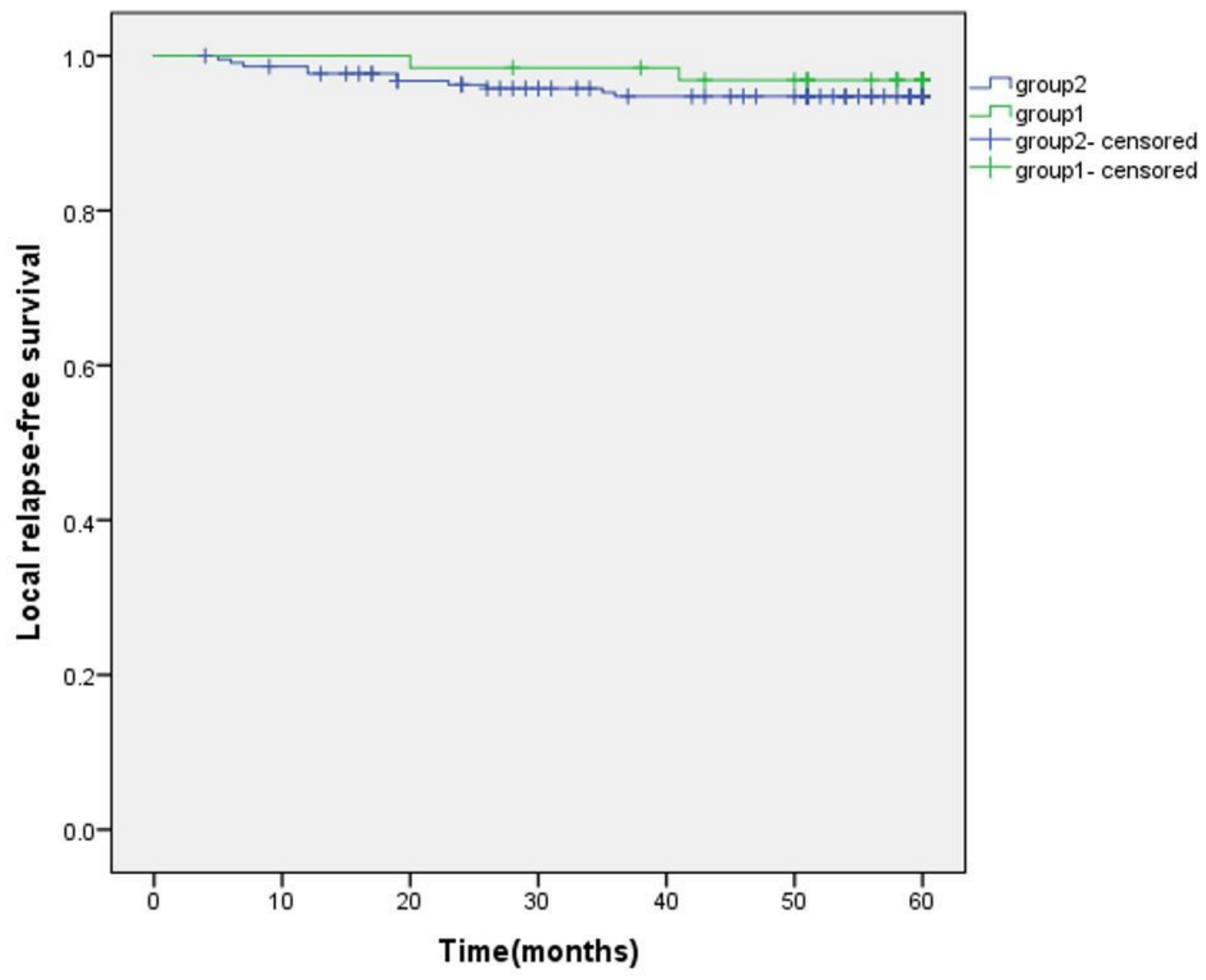

Figure 3

Kaplan-Meier estimate of LRFS for patients who accepted capecitabine maintenance chemotherapy \group 1囚and patients who did not accept maintenance chemotherapy \group 2】. 


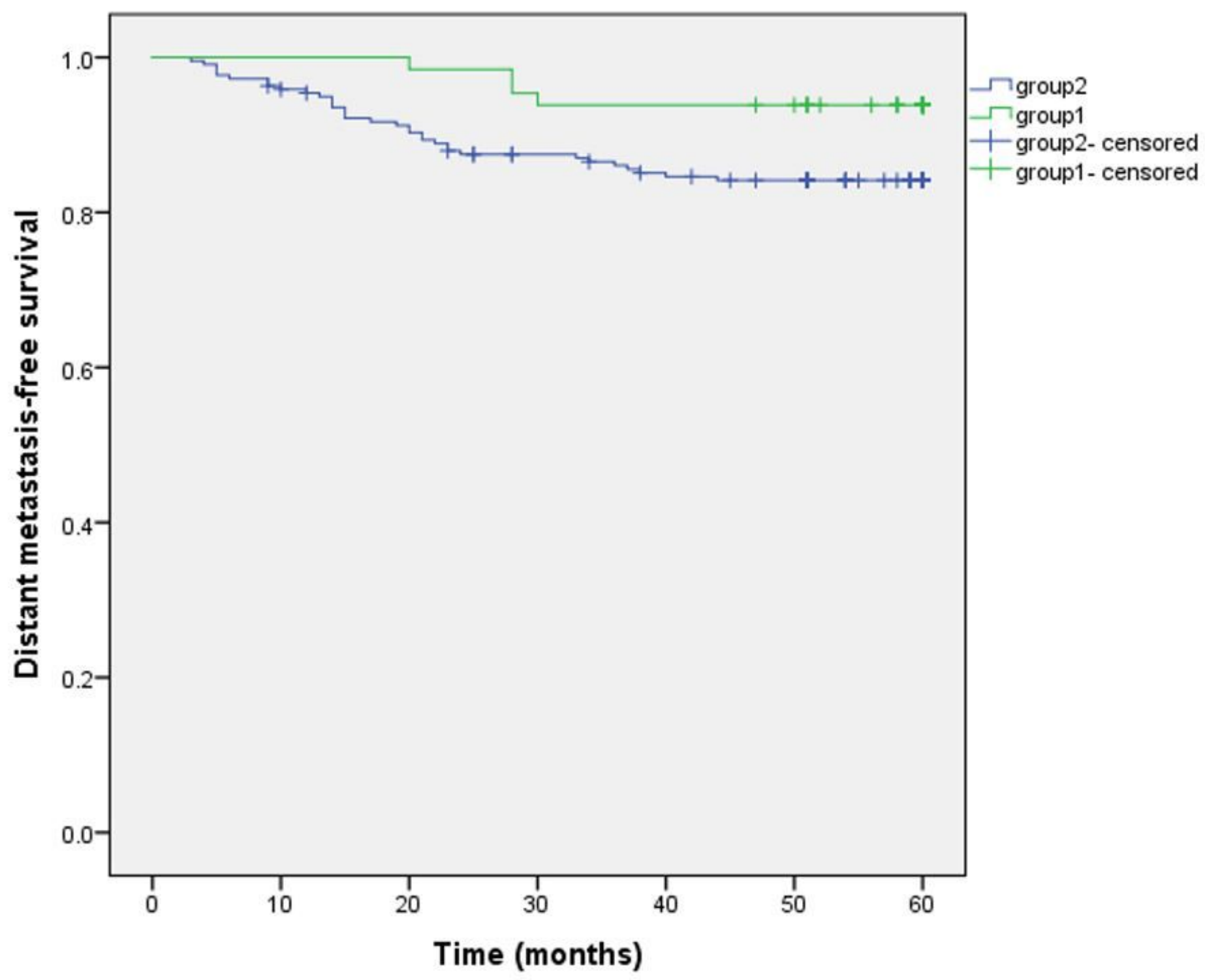

Figure 4

Kaplan-Meier estimate of DMFS for patients who accepted capecitabine maintenance chemotherapy 囚group 1囚and patients who did not accept maintenance chemotherapy \group 2】. 